KYUNGPOOK Math. J. 55(2015), 191-203

http://dx.doi.org/10.5666/KMJ.2015.55.1.191

pISSN 1225-6951 eISSN 0454-8124

(C) Kyungpook Mathematical Journal

\title{
On a Quasitoric Virtual Braid Presentation of a Virtual Link
}

\author{
Yonguu BaE* and Seogman Seo \\ Department of Mathematics, Kyungpook National University, Daegu 702-701, Korea \\ e-mail: ybae@knu.ac.kr and seogmanseo@knu.ac.kr
}

ABSTRACT. We introduce a quasitoric virtual braid and show that every virtual link can be obtained by the closure of a quasitoric virtual braid. Also, we show that the set of quasitoric virtual braids with $n$ strands forms a group which is a subgroup of the $n$-virtual braid group.

\section{Introduction}

The notion of a quasitoric braid is introduced by Manturov[7] as a generalization of a toric braid which can be drawn on the standard torus, and he proved that every link can be presented as the closure of a quasitoric braid. By the virtue of Manturov's theorem, one can define the quasitoric braid index of a link $L$ as the minimum number of strands of a quasitoric braid which presents the given link $L$. In [1], the authors gave a formula to calculate the quasitoric braid index.

The concept of a virtual link was proposed by Kauffman[4] as a generalization of the notion of a classical link. Kamada[3] proved that any virtual link can be presented by the closure of a virtual braid which is unique up to certain basic moves. The result is analogous to Alexander theorem and Markov theorem for classical braids and links. Kauffman and Lambropoulou[6] proved the same result by using $L$-moves.

In this paper, we introduce the notion of a quasitoric virtual braid, and prove that every virtual link can be presented as the closure of a quasitoric virtual braid.

* Corresponding Author.

Received January 19, 2015; accepted February 27, 2015.

2010 Mathematics Subject Classification: 57M25, 57M27.

Key words and phrases: Link, Knot, Braid, Toric Braid, Quasitoric braid, Braid index, Quasitoric braid index.

This work was supported by Basic Science Research Program through the National Research Foundation of Korea (NRF) funded by the Ministry of Education, Science and Technology (2013R1A1A4A01009028). 


\section{Virtual Links and Virtual Braids}

A virtual link diagram is a link diagram in $\mathbb{R}^{2}$ possibly with encircled crossings without over/under information, see Figure 1. Such an encircled crossing is called a virtual crossing.

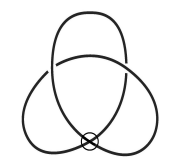

Figure 1: Virtual knot

Two virtual link diagrams are said to be equivalent if one can be transformed into another by applying finite times of the generalized Reidemeister moves which consist of classical Reidemeister moves $R_{1}, R_{2}$ and $R_{3}$ and virtual Reidemeister moves $V_{R_{1}}, V_{R_{2}}, V_{R_{3}}$ and the semi-virtual move $V_{R_{4}}$ as shown in Figure 2. An equivalence class of a virtual link diagram is called a virtual link.
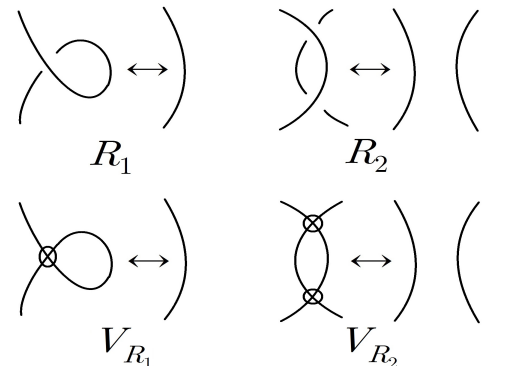
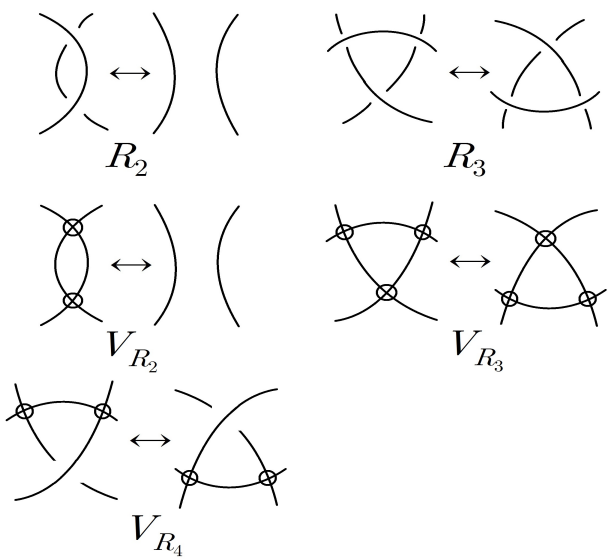

Figure 2: Generalized Reidemeister moves

A succinct depiction of virtual equivalence is that it is generated by classical Reidemeister moves and the detour move which is shown in Figure 3. 


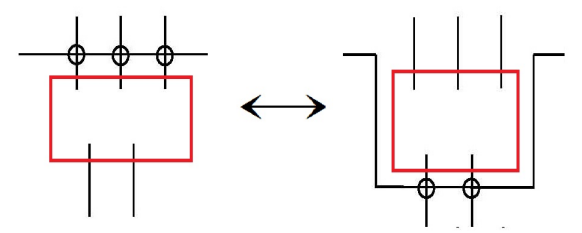

Figure 3: The detour move

One can define the virtual braid group $\mathbf{v} \mathbf{B}_{n}$ as an extension of the classical braid group $\mathbf{B}_{n}$.

Definition 2.1.([3]) Let $n$ be a positive integer and $P_{n}=\left\{x_{i} \mid x_{1}<x_{2}<\cdots<x_{n}\right\}$ a set of $n$ interior points of the interval $[0,1]$. Define $E$ in $\mathbb{R}^{2}$ by $[0,1] \times[0,1]$ and $\pi_{2}: E \longrightarrow[0,1]$ by the second factor projection. A virtual braid diagram with $n$ strands is an immersed 1-manifold $b=a_{1} \cup \cdots \cup a_{n}$ in $E$ such that

(1) $\partial b=P_{n} \times\{0,1\} \subset E$,

(2) for each $i=1, \cdots, n,\left.\pi_{2}\right|_{a_{i}}: a_{i} \longrightarrow[0,1]$ is a homeomorphism,

(3) the multiple point set $V(b)$ consists of transverse double points,

(4) $\left.\pi_{2}\right|_{V(b)}: V(b) \longrightarrow[0,1]$ is injective,

(5) each point of $V(b)$ is assigned an information of classical crossing or virtual crossing as shown in Figure 4.

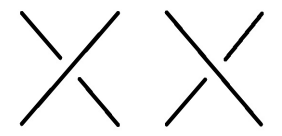

Classical crossings

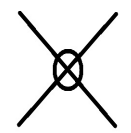

Virtual crossing

Figure 4: Crossings

Two virtual braid diagrams said to be equivalent if one is transformed to the other continuously keeping the above conditions. An equivalence class of a virtual braid diagram with $n$ strands is called a $n$-virtual braid. The set of $n$-virtual braid, with the concatenation product, forms a monoid which is generated by $\sigma_{i}, \sigma_{i}^{-1}$ and $v_{i}$, in Figure 5, for $i=1,2, \cdots, n-1$. 

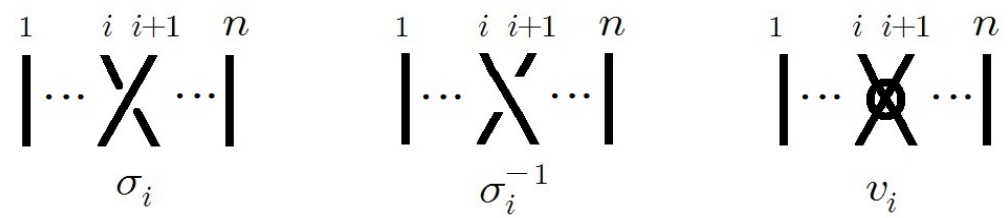

Figure 5: Generators

Definition 2.2.([3]) The virtual braid group $\mathbf{v B}_{n}$ is the group obtained from the monoid of $n$-virtual braids by introducing the following relations:

$$
\begin{array}{ll}
\sigma_{i} \sigma_{i}^{-1}=\sigma_{i}^{-1} \sigma_{i}=1, \quad v_{i}^{2}=1 & \\
\sigma_{i} \sigma_{j}=\sigma_{i} \sigma_{j}, \quad v_{i} v_{j}=v_{j} v_{i} & ,|i-j| \geq 2 \\
\sigma_{i} \sigma_{i+1} \sigma_{i}=\sigma_{i+1} \sigma_{i} \sigma_{i+1} & \\
v_{i} v_{i+1} v_{i}=v_{i+1} v_{i} v_{i+1} & ,|i-j| \geq 2 \\
\sigma_{i} v_{j}=v_{j} \sigma_{i} & \\
v_{i} \sigma_{i+1} v_{i}=v_{i+1} \sigma_{i} v_{i+1} &
\end{array}
$$

In [5], Kauffman and Lambropoulou introduced the following relation,

$$
\sigma_{i+1}=\left(v_{i} \cdots v_{2} v_{1}\right)\left(v_{i+1} \cdots v_{3} v_{2}\right) \sigma_{1}\left(v_{2} v_{3} \cdots v_{i+1}\right)\left(v_{1} v_{2} \cdots v_{i}\right)
$$

for each $i=1, \cdots, n-2$. In terms of $n$-virtual braids, this relation is a consequence of the above defining relations and it called the braid detour moves of the strands $1,2, \cdots, i$, around the crossing $\sigma_{i+1}$. See Figure 6.

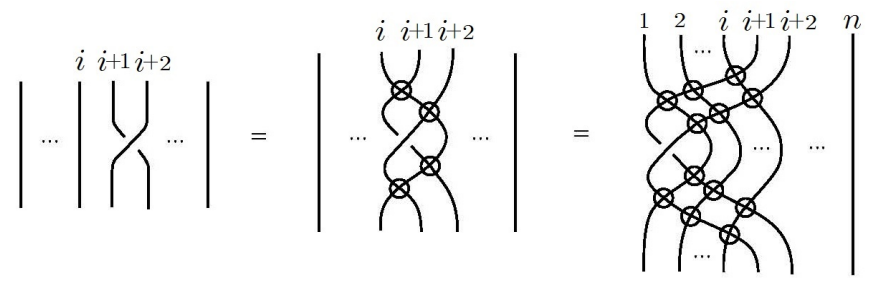

Figure 6: Detouring the crossing $\sigma_{i+1}$

Since the strands of an $n$-virtual braid $\beta$ connect injectively the points in $\left\{p_{i} \mid p_{i}=\left(x_{i}, 1\right), x_{i} \in P_{n}\right\}$ to the points in $\left\{q_{i} \mid q_{i}=\left(x_{i}, 0\right), x_{i} \in P_{n}\right\}$ for an $n$-virtual braid $\beta$, there is an element $\pi(\beta)$ in the permutation group $\mathbf{S}_{n}$ which is uniquely determined by each strand. Indeed, if the $k$ th strand of $\beta$ connects $p_{i}$ to $q_{i_{k}},(k=1,2, \cdots, n)$, then the permutation $\pi(\beta)$ corresponding to $\beta$ is 
$\left(\begin{array}{cccc}1 & 2 & \cdots & n \\ i_{1} & i_{2} & \cdots & i_{n}\end{array}\right)$. For example, the permutation corresponding to $\beta$ in Figure 7 (a) is $\left(\begin{array}{llll}1 & 2 & 3 & 4 \\ 3 & 1 & 4 & 2\end{array}\right)$.

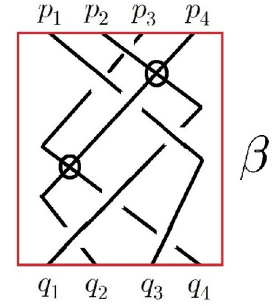

(a)

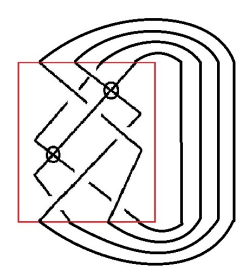

(b)

Figure 7: A virtual braid and its closure

A virtual braid is said to be pure if its permutation is the identity permutation. The closure $\widehat{\beta}$ of a virtual braid $\beta$ is the virtual link obtained by joining the points $p_{1}, p_{2}, \cdots, p_{n}$ to the points $q_{1}, q_{2}, \cdots, q_{n}$ by parallel curves as shown in Figure 7 (b).

Proposition 2.3 and Proposition 2.4 were proved by Kamada which are the analogues of Alexander theorem and Markov theorem respectively.

Proposition 2.3.([3]) Virtual links can be represented by the closures of virtual braids.

Proposition 2.4.([3]) Two virtual braid diagrams have equivalent closures as virtual links if and only if they are related to each other by a finite sequence of the following VM moves (1) - (4).

(1) (VM0-move) a virtual braid move.

(2) (VM1-move) a conjugation in the virtual braid group.

(3) (VM2-move) a right stabilization of positive, negative or virtual crossing and its invrse operation, see Figure 8.

(4) (VM3-move) a right/left virtual exchange moves, see Figure 9.

VM0-move, VM1-move and VM2-move are analogous to those in the classical case and the new move has two variants, the right one and the left one. It is remarkable that the right VM3-move is not consequences of the left VM3-move, vice versa. Two virtual braids are VM-equivalent if they are related by a finite sequence of VM moves which are mentioned in Proposition 2.4. 


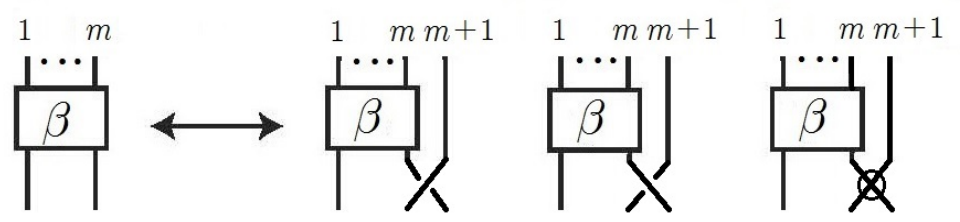

Figure 8: Right stabilizations/destabilizations
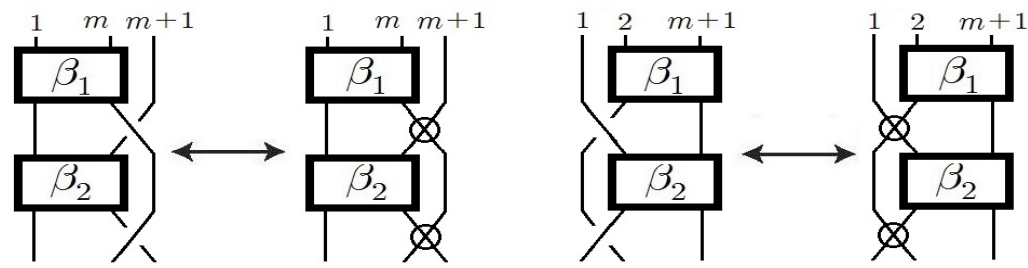

Figure 9: Right/left exchange moves

\section{Quasitoric Virtual Braids}

We introduce the definition of a quasitoric virtual braid.

Definition 3.1. Let $p$ and $q$ be positive integers with $p \geq 2$. A virtual braid $\beta$ is said to be quasitoric of type $(p, q)$ if it can be expressed as $\beta_{1} \beta_{2} \cdots \beta_{q}$ where for each $\beta_{j}=x_{p-1}^{e_{j, p-1}} \cdots x_{1}^{e_{j, 1}}$ where $x_{i} \in\left\{\sigma_{i}, v_{i}\right\}$ and each $e_{j, i}$ is either 1 or -1 if $x_{i}=\sigma_{i}$ and $e_{j, i}=1$ if $x_{i}=v_{i}$, see Figure 10. Shortly, a quasitoric braid of type $(p, q)$ is called a p-quasitoric virtual braid.

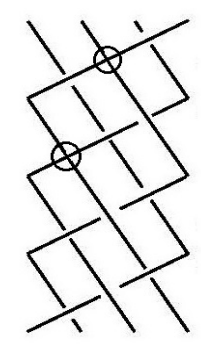

Figure 10: Example of a quasitoric virtual braid 
Lemma 3.2. Let $\beta$ be a quasitoric virtual braid of type $(p, q)$. If $\beta$ is a pure virtual braid, then $q=p \cdot k$ for some positive integer $k$.

Proof. Let $\beta=\beta_{1} \beta_{2} \cdots \beta_{q}$ where for each $\beta_{j}=x_{p-1}^{e_{j, p}-1} \cdots x_{1}^{e_{j, 1}}$ where $x_{i} \in\left\{\sigma_{i}, v_{i}\right\}$ and each $e_{j, i}$ is either 1 or -1 if $x_{i}=\sigma_{i}$ and $e_{j, i}=1$ if $x_{i}=v_{i}$. Then $\pi\left(\beta_{j}\right)=$ $(12 \cdots p)$ for all $j$ and the order of $(12 \cdots p)$ is $p$. Since $\beta$ is a pure virtual braid, $\pi(\beta)$ is the identity permutation. Since $\pi(\beta)=\pi\left(\beta_{1} \beta_{2} \cdots \beta_{q}\right)=\pi\left(\beta_{1}\right) \pi\left(\beta_{2}\right) \cdots \pi\left(\beta_{q}\right)=$ $(12 \cdots q)^{q}, p$ divides $q$. Hence $q=p \cdot k$ for some positive integer $k$.

Also, we introduce the definition of a $(i, j)$-quasitoric virtual braid.

Definition 3.3. For positive integers $i, j$ with $1 \leq i<j \leq p$, a $p$-strand virtual braid $\beta$ is called a $(i, j)$-quasitoric virtual braid with $p$ strands if it has a virtual braid diagram of the form in Figure 11, where $\gamma$ is a $(j-i+1)$-quasitoric virtual braid.

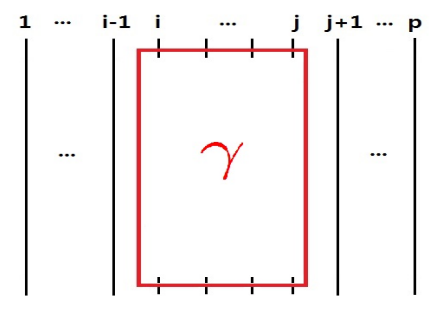

Figure 11: $(i, j)$-quasitoric virtual braid with $p$ strands

Note that if a $(i, j)$-quasitoric virtual braid $\beta$ is pure, then the corresponding $(j-i+1)$-quasitoric virtual braid $\gamma$ is a quasitoric pure virtual braid of type $(j-i+1,(j-i+1) k)$ for some positive integer $k$.

Theorem 3.4. For integer $i, j$ with $1 \leq i<j \leq p$, every $(i, j)$-quasitoric pure virtual braid with $p$ strands is quasitoric.

Proof. For a positive integer $p$ with $p \geq 3$, it suffice to show that $(1, p-1)$ quasitoric pure virtual braid and $(2, p)$-quasitoric pure virtual braid with $p$ strands are quasitoric. Consider a $(p-1)$-quasitoric pure virtual braid diagram $\gamma$ which lies in a $(1, p-1)$-quasitoric pure virtual braid diagram $D$ with $p$ strands. We slide the $p$ th vertical strand into $\gamma$ such that the $p$ th strands is parallel with the $(p-1)$ th strand by using detour moves as shown in Figure 12. No sooner than the sliding of the $p$ th strand, we can pass the section of the $p$ th strand with positive slope over the $(p-1)$ th strand by using detour moves as shown in Figure 13. Then we obtain a $p$ quasitoric pure virtual braid diagram which is equivalent to the $(1, p-1)$-quasitoric pure virtual braid diagram with $p$ strands. Similarly, we can make a $p$-quasitoric pure virtual braid diagram which is equivalent to the $(2, p)$-quasitoric pure virtual 


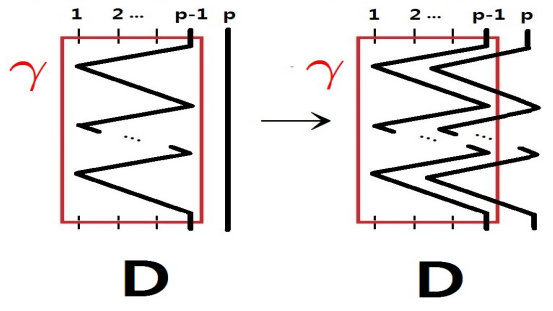

Figure 12: Slide the $p$ th strand into $\gamma$

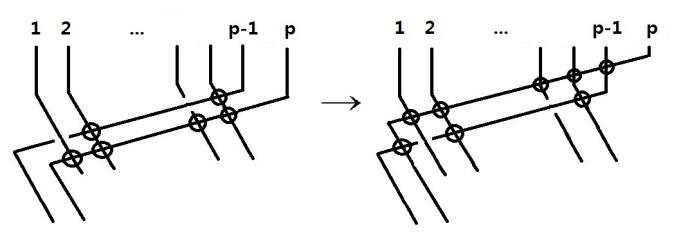

Figure 13: the $p$ th strand pass over the $(p-1)$ th strand

braid diagram with $p$ strands. By using this process inductively, we have a quasitoric pure virtual braid diagram of a $(i, j)$-quasitoric pure virtual braid.

For example, we can make a quasitoric pure virtual braid diagram of a $(1,4)$ quasitoric pure virtual braid with 5 strands as shown in Figure 14.

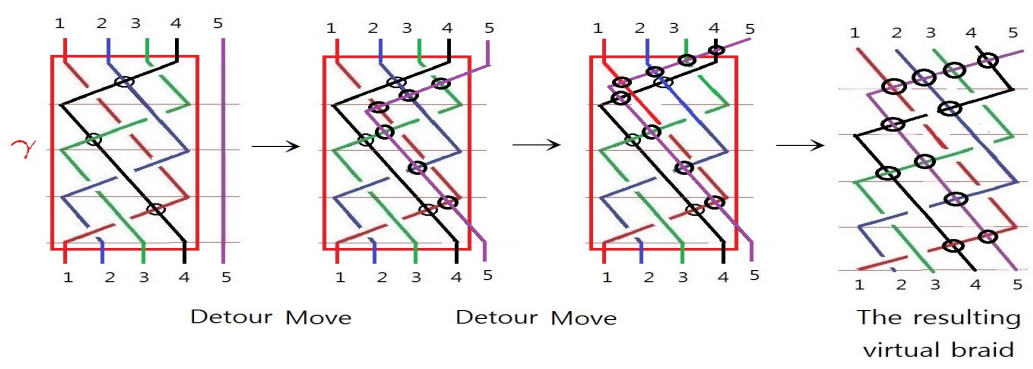

Figure 14: The resulting virtual braid 
In [2], Bardakov introduced a presentation of the pure virtual braid group $\mathbf{P v B}_{n}$ with $n$-strands as the following.

$$
P v B_{n}=\left\langle R_{i j} \mid R_{i j} R_{i k} R_{j k}=R_{j k} R_{i k} R_{i j}, R_{i j} R_{k l}=R_{k l} R_{i j}\right\rangle,
$$

where $1 \leq i \neq j \leq n$, the generators $R_{i j}$ and $R_{j i}$ are shown in Figure 15 .

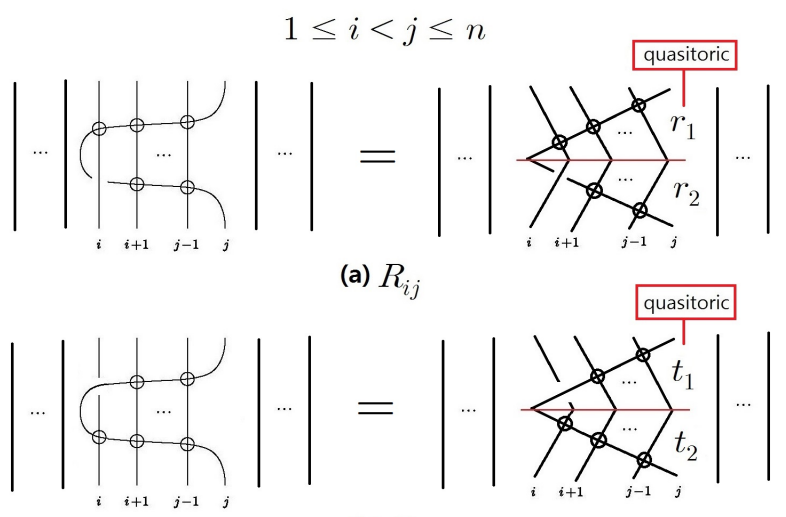

(b) $R_{j i}$

Figure 15: Generators $R_{i j}$ and $R_{j i}$

Theorem 3.5. Every pure virtual braid is quasitoric.

Proof. It suffices to show that the generators $R_{i j}$ and $R_{j i}$ can be presented as a quasitoric virtual braid. $R_{i j}$ and $R_{j i}$ can be deformed to the diagrams in the right of Figure 15. Note that $r_{1}$ and $t_{1}$ in Figure 15 are quasitoric. Firstly, we will show that $R_{i j}$ is quasitoric.

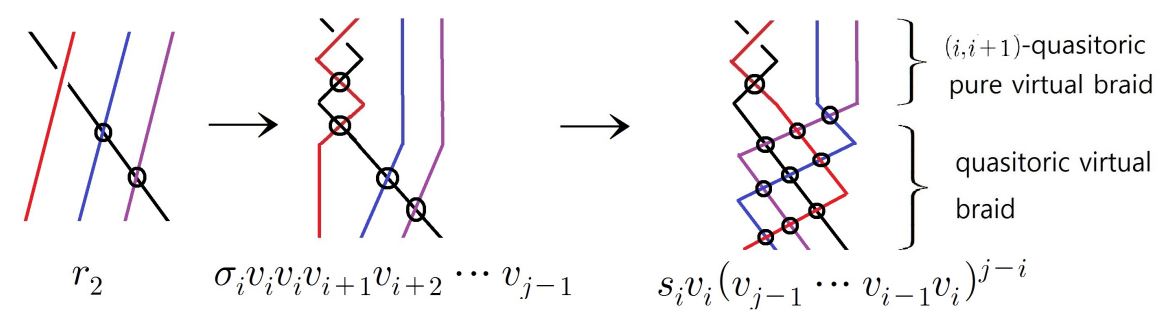

Figure 16: Make $r_{2}$ as a quasitoric virtual braid 
Note that the braid word of $r_{2}$ is $\sigma_{i} v_{i+1} v_{i+2} \cdots v_{j-1}$. By using the following equivalence:

$$
\begin{aligned}
& \sigma_{i} v_{i+1} v_{i+2} \cdots v_{j-1} \\
\simeq & \sigma_{i} v_{i} v_{i} v_{i+1} v_{i+2} \cdots v_{j-1} \\
\simeq & \sigma_{i} v_{i}\left(v_{j-1} \cdots v_{i-1} v_{i}\right)^{j-i}
\end{aligned}
$$

as shown in Figure 16, we can make $r_{2}$ as the product of a $(i, i+1)$-quasitoric pure virtual braid with $(j-i+1)$ strands and a quasitoric virtual braid. Since we already show that a $(i, j)$-quasitoric pure virtual braid is quasitoric in Theorem 3.4, we can present $r_{2}$ as a quasitoric virtual braid.

$R_{i j}^{-1}$ is depicted in Figure 17 (a), and we can deform $R_{i j}^{-1}$ to the diagram in the right of Figure 17 (b).

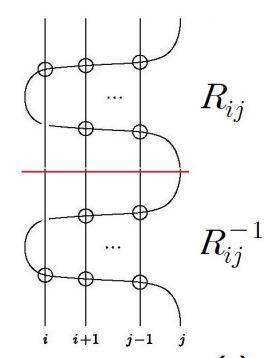

(a)

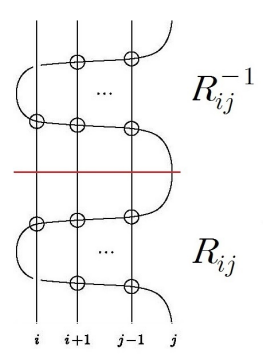

$i_{i+1} \quad j-1 \quad j$

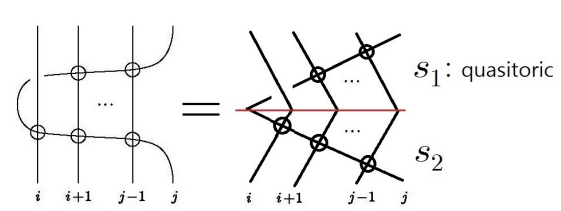

(b)

Figure 17: $R_{i, j}^{-1}$

Note that the braid word of $s_{2}$ is $v_{i} v_{i+1} v_{i+2} \cdots v_{j-1}$. In a similar manner of $r_{2}$, we can make $s_{2}$ as a quasitoric virtual braid by using the following equivalence:

$$
v_{i} v_{i+1} v_{i+2} \cdots v_{j-1} \simeq\left(v_{j-1} \cdots v_{i-1} v_{i}\right)^{j-i} .
$$

Since $s_{2}$ is a quasitoric virtual braid, $R_{i j}^{-1}$ can be presented as a $(i, j)$-quasitoric pure virtual braid. By Theorem 3.4, $R_{i j}^{-1}$ is quasitoric. Similarly, we can show that $R_{j i}$ and $R_{j i}^{-1}$ are quasitoric also. Therefore every pure virtual braid is quasitoric.

In [3], Kamada showed that any virtual link can be presented by the closure of a virtual braid $\beta$. In order to obtain a quasitoric virtual braid presentation of $\beta$, we must transform $\beta$ in a proper way.

Theorem 3.6. Every virtual link can be obtained as the closure of a quasitoric virtual braid.

Proof. For a given virtual link $L$, there exist a virtual braid $\beta$ such that the closure of $\beta$ is equivalent to $L$. Suppose that $\beta$ is a virtual braid with $n$ strands and $\pi(\beta)$ is the permutation of $\beta$. Now consider orbits of the action of $\pi(\beta)$ on the set $\{1,2, \cdots, n\}$. 
Then these orbits contain different numbers of elements. Let us apply the VM2move and the VM3-move for transforming these orbits. The VM2-move conjugates the braid. So, the number of elements in orbits does not change, but elements in orbits are permuted. Because, it conjugates the corresponding permutation. The VM3-move increases the number of strands by one, since it adds the element $n+1$ to the orbit which is containing the element $n$ and does not change other orbits. Thus, by using the VM2-move and the VM3-move, one can re-enumerate elements in such a way that the smallest orbit by one. Moreover, repeating this many times, we can obtain a virtual braid $\tau$ with the same number of elements for all orbit. Now suppose that the number of strands of $\tau$ is $k m$ for positive integers $k$ and $m$. By conjugating $\tau$, we can get a virtual braid $\alpha$ such that the corresponding permutation of $\alpha$ is the $r$ th power of the cyclic permutation $(12 \cdots \mathrm{km})$ for positive integer $r$. Then the resulting virtual braid $\alpha$ is VM-equivalent to $\beta$. Let $\kappa=\sigma_{k m-1} \sigma_{k m-2} \cdots \sigma_{2} \sigma_{1}$, then $\alpha \kappa^{-r}$ is a pure virtual braid. By using Theorem 3.5, $\alpha \kappa^{-r}$ is quasitoric and put $\alpha \kappa^{-r}=\delta$. Then we have

$$
\alpha=\delta \kappa^{r} .
$$

Note that the product of two $n$-quasitoric virtual braids is a $n$-quasitoric virtual braid. Since $\kappa$ and $\delta$ are quasitoric, $\alpha$ is quasitoric. Therefore there exist a quasitoric virtual braid $\alpha$ such that $\alpha$ is quasitoric and VM-equivalent to $\beta$. It means that $\alpha$ is a quasitoric presentation of the given link $L$.

Now we will show that the set $\mathbf{Q v B}_{n}$ of $n$-quasitoric virtual braids forms a group which is a subgroup of the $n$-virtual braid group.

Theorem 3.7. Let $\mathbf{Q v B} \mathbf{B}_{n}$ be the set of n-quasitoric virtual braids. Then $\mathbf{Q v B}_{n}$ is a subgroup of the virtual braid group $\mathbf{v B}_{n}$.

Proof. It suffices to show that the inverse of a $n$-quasitoric virtual braid in $\mathbf{v B}_{n}$ is quasitoric, for every positive integer $n$. Let $\beta=x_{n-1}^{e_{n-1}} x_{n-2}^{e_{n-2}} \cdots x_{2}^{e_{2}} x_{1}^{e_{1}}$ where $x_{i} \in\left\{\sigma_{i}, v_{i}\right\}$ and each $e_{i}$ is either 1 or -1 if $x_{i}=\sigma_{i}$ and $e_{i}=1$ if $x_{i}=v_{i}$ for positive integer $n$, we have to prove that there exist a quasitoric virtual braid $\delta$ such that $\beta \cdot \delta$ and $\delta \cdot \beta$ are the trivial. first put $\delta$ as $x_{1}^{d_{1}} x_{2}^{d_{2}} \cdots x_{n-2}^{d_{n-2}} x_{n-1}^{d_{n-1}}$ each $d_{i}=-e_{i}$ if $x_{i}=\sigma_{i}$ and $d_{i}=1$ if $x_{i}=v_{i}$ then by using two relations $\sigma \sigma^{-1}=\sigma^{-1} \sigma=1$ and $v_{i} v_{i}=1$, we have the following:

$$
x_{n-1}^{e_{n-1}} x_{n-2}^{e_{n-2}} \cdots x_{2}^{e_{2}} x_{1}^{e_{1}} x_{1}^{d_{1}} x_{2}^{d_{2}} \cdots x_{n-2}^{d_{n-2}} x_{n-1}^{d_{n-1}}=1
$$

and

$$
x_{1}^{e_{1}} x_{1}^{d_{1}} x_{2}^{d_{2}} \cdots x_{n-2}^{d_{n-2}} x_{n-1}^{d_{n-1}} x_{n-1}^{e_{n-1}} x_{n-2}^{e_{n-2}} \cdots x_{2}^{e_{2}} x_{1}^{e_{1}}=1 .
$$

Hence $\delta$ is the inverse of $\beta$, see Figure 18. We will show that $\delta$ is quasitoric. To begin with, we will product a trivial quasitoric virtual braid to the given inverse, as shown in Figure 19 (b). Then we can see that the resulting virtual braid is presented by a product of two virtual braids, see Figure 19 (b). Moreover, we can see that one of two braids is a pure virtual braid and the other is a quasitoric virtual braid. In Theorem 3.5, we show that every pure virtual braid is quasitoric, therefore we can 

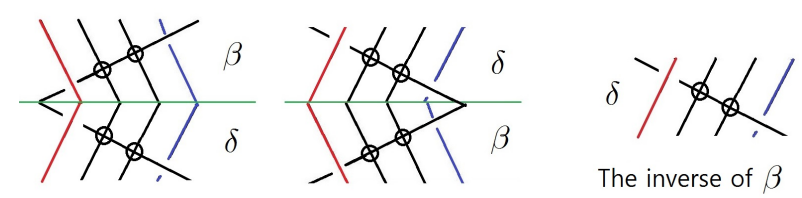

Figure 18: Generator and its inverse

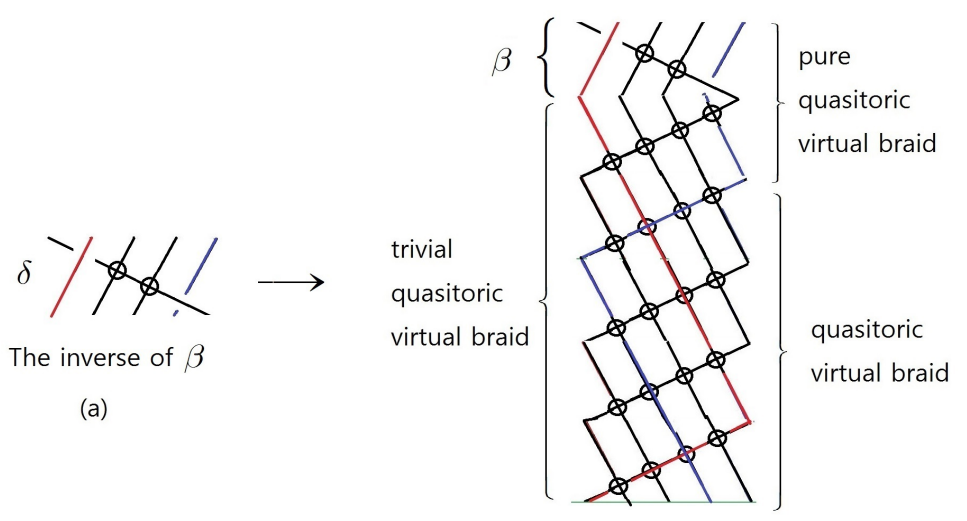

(b)

Figure 19: Quasitoric presentation of $\delta$

make $\delta$ as a quasitoric virtual braid. Since every quasitoric virtual braid is generated by $\beta$, we can show that the inverse of a given quasitoric virtual braid is quasitoric.

\section{References}

[1] Y. Bae and S. Seo, On the quasitoric braid index of a link, preprint (2014).

[2] V. G. Bardakov, The virtual and universal braids, Fund. Math., 184(2004), 1-18.

[3] S. Kamada, Braid presentation of virtual knots and welded knots, Osaka J. Math., 44(2007), 441-458.

[4] L. H. Kauffman, virtual knot theory, European J. Combin., 20(1999), 663-690.

[5] L. H. Kauffman and S. Lambropoulou, Virtual braids, Fund. Math., 184(2004), 159186. 
[6] L. H. Kauffman and S. Lambropoulou, Virtual braids and L-moves, J. Knot Theory Ramifications, 15(2006), 773-811.

[7] V. O. Manturov, A combinatorial representation of links by quasitoric braids, European J. Combin., 23(2002), 207-212. 